\title{
Original Paper / Artigo Original Riparian vegetation structure and soil variables in Pandeiros river, Brazil
}

\author{
Nathalle Cristine Alencar Fagundes ${ }^{1,6}$, Marly Antonielle de Ávila ${ }^{2}$, Saimo Reblleth de Souza ${ }^{3}$, \\ Islaine Franciely Pinheiro de Azevedo ${ }^{3}$, Yule Roberta Ferreira Nunes ${ }^{3}$, Geraldo Wilson Fernandes ${ }^{4}$, \\ Luiz Arnaldo Fernandes ${ }^{5}$, Rubens Manoel dos Santos $^{2}$ \& Maria das Dores Magalhães Veloso ${ }^{3}$
}

\begin{abstract}
This study analyzed the floristic, structural variations and their relationships with soil variables in two stretches of riparian vegetation in an ecotonal region between savanna and semiarid zones. We following the hypothesis of despite the proximity between the sampled areas, soil conditions are distinct and lead to changes in structure and composition of the vegetation. This study was development at EPA of Pandeiros River, Minas Gerais, where we allocated 140 plots of $10 \times 10 \mathrm{~m}\left(100 \mathrm{~m}^{2}\right)$, with $10 \mathrm{~m}$ distance between plots, in two areas of riparian vegetation, named Larga and São Domingos (70 plots in each area), where we conducted the phytosociological survey and collected soil samples. In total, 751 arboreal individuals were sampled, distributed in 89 species and 35 botanical families. The areas differed strongly in structure and species composition, and showed floristic peculiarities and influence of surrounding vegetation. Despite of low distance between the sampled areas, these are singular environments influenced by different soils, by the mixed composition of the ecotonal area and the anthropogenic impacts to which they are exposed.

Key words: diversity, ecological corridors, floristic, phytosociology, environmental filter.

Resumo

Este estudo analisou as variações florísticas, estruturais e suas relações com variáveis do solo em dois trechos de vegetação ripária em uma região ecotonal entre savana e zonas semi-áridas. Propomos a hipótese de que, apesar da proximidade entre as áreas amostradas, as condições do solo são distintas e levam a mudanças na estrutura e composição da vegetação, mas essas mudanças também são influenciadas pelo caráter ecotonal. Este estudo foi desenvolvido na APA do rio Pandeiros, Minas Gerais, onde alocamos 140 parcelas de $10 \times 10$ m $\left(100 \mathrm{~m}^{2}\right)$, com $10 \mathrm{~m}$ de distância entre parcelas, em duas áreas de vegetação ripária denominadas Larga e São Domingos (70 parcelas em cada área), onde realizamos o levantamento fitossociológico e coletamos amostras de solo. No total, 751 indivíduos arbóreos foram amostrados, distribuídos em 89 espécies e 35 famílias botânicas. As áreas diferiram fortemente na estrutura e composição das espécies, e apresentaram peculiaridades florísticas e influência da vegetação circundante. Apesar da baixa distância entre as áreas amostradas, estes são ambientes singulares influenciados por diferentes solos, pela composição mista da área ecotonal e os impactos antropogênicos a que estão expostas.
\end{abstract}

Palavras-chave: diversidade, corredores ecológicos, florística, fitossociologia, filtro ambiental.

\section{Introduction}

The riparian vegetation plays an important role in maintaining the integrity of local ecosystems and is vital to protection of waterways and associated fauna (Bennett et al. 2014; Dietrich et al. 2015). Despite its importance, the human action has transformed these forest corridors in isolated fragments, causing disruption of many ecological services and biodiversity loss (Corenblit et al. 2015), making the riparian forests the most

\footnotetext{
${ }^{1}$ Universidade Federal de Lavras, Depto. Biologia, Campus Universitário, CP 303, 37200-000, Lavras, MG, Brazil.

${ }^{2}$ Universidade Federal de Lavras, Depto. Ciências Florestais, Campus Universitário, CP 3037, 37200-000 Lavras, MG, Brazil.

${ }^{3}$ Universidade Estadual de Montes Claros, Depto. Biologia Geral, Laboratório de Ecologia Vegetal, 39401-089, Montes Claros, MG, Brazil.

${ }^{4}$ Universidade Federal de Minas Gerais, Depto. Biologia Geral, 31270-901, Belo Horizonte, MG, Brazil.

${ }^{5}$ Universidade Federal de Minas Gerais, Inst. Ciências Agrárias, 39404-547, Montes Claros, MG, Brazil.

${ }^{6}$ Author for correspondence: nath_fag@yahoo.com.br
} 
degraded ecosystems in the world (Dietrich et al. 2015). In face of anthropogenic pressures, floristic and structural studies are needed, as broaden the knowledge of a particular community, which allows to trace the profile of habitats with specific environmental characteristics (Corenblit et al. 2015). When to addressed abiotic factors, such as edaphic attributes, these studies provides, besides floristic composition, quality of environment, structure and history of vegetation, information in order to understand how these abiotic factors can influence the communities of these landscapes (Caiafa \& Martins 2007). Arruda et al. (2013) show that edaphic factors have a strong role in vegetation at local scale, establishing subgroups in vegetal formations with separated affinities.

The riparian vegetation is strongly influenced by local factors, such as soil and topographical, and by adjacent vegetation, resulting in a mixed floristic composition, with species typical of river banks and other phytophisiognomies (Azevedo et al. 2014; Veloso et al. 2014). Also, due to high frequency of changes mainly by flooding regime, natural and anthropogenic disturbances, riparian vegetation usually has a high variation in structure and spatial distribution of their species (Rodrigues et al. 2009; Veloso et al. 2014). Thus, these forests concentrate a high richness of forest species, which tends to be superior than vegetation in non-riparian areas (Bennett et al. 2014).

Among the factors that influence riparian vegetation structure, the soil is a major contributor to distribution and diversity of flora (Arruda et al. 2013; Lloyd et al. 2015). Some studies have found different responses on floristic composition in relation to the type of soil and water availability (Rossi et al. 2005; Arruda et al. 2013; Lloyd et al. 2015). Some soil parameters such as texture, depth, nutrients disponibility and toxicity elements have signed a direct relation with vegetation (Rossi et al. 2005). The studies that seek to establish a correlation between vegetation and habitat are highly importance, because those can be a determinant of the distribution and diversity of species in an area (Rossi et al. 2005; Arruda et al. 2013).

In the transition areas, riparian vegetation presents complex combinations of species components of neighbors' phytogeographic domains, with a range of different plant species with high specificity and adaptation to environmental heterogeneity (Azevedo et al. 2014; Bennett et al. 2014; Corenblit et al. 2015). This differentiation is greater in arid or semi-arid environments, where riparian vegetation can act as an ecological corridor, sheltering more richness and abundance than adjacent areas (Bennett et al. 2014; Corenblit et al. 2015). The north of Minas Gerais is a semiarid ecotonal region between Caatinga (shrub and dry forests) and Cerrado (brazilian savanna), and thus comprises a mosaic where the riparian vegetation may, in addition to characteristic species of this unit, typical species of phytogeographic units outside the riparian matrix (Azevedo et al. 2014; Veloso et al. 2014). The name "riparian vegetation" is coined in the present study for it is an ecotone area where species of different vegetation areas are present, fleeing the composition commonly found in riparian forests.

Information about species composition, vegetation structure and interactions between vegetation and edaphic factors is needed also for developing successful restoration projects in threatened areas with peculiar physiognomy (Madeira et al. 2009). Thus, we analyze floristic and structural variations and their relationships with soil variables in two stretches of riparian vegetation in north of Minas Gerais. For this purpose, we following the hypothesis of despite the proximity between sampled areas, soil conditions are distinct and lead to changes in structure and composition of riparian vegetation.

\section{Material and Methods}

Study area

The study was developed in the Environmental Protection Area of Pandeiros River (EPA Pandeiros River), covering the municipalities of Januária, Cônego Marinho and Bonito de Minas, northern region of Minas Gerais, Brazil (Fig. 1). The EPA of Pandeiros river presents Cerrado sensu strictu as predominant physiognomy, but seasonally dry tropical forest and palm swamps are very common in the area, forming a complex riparian vegetation with mixed floristic composition and strongly influenced by these physiognomies (Nunes et al. 2009; Azevedo et al. 2014).

Soils are classified as Quartzarenic Neosol, with notoriously sandy texture, Dystrophic Haplic Cambisol, unstable predominantly silt and Latosol with high water storage capacity, acid and low fertility, and Gleysols are distributed by the linear relief surfaces in palm swamps (Embrapa 2013). The Koppen's climate classification is tropical type (Aw), with rainy and dry seasons well defined, dry winter and rainy summer, where the average annual rainfall varies from 900 to $1,200 \mathrm{~mm}$ and the 


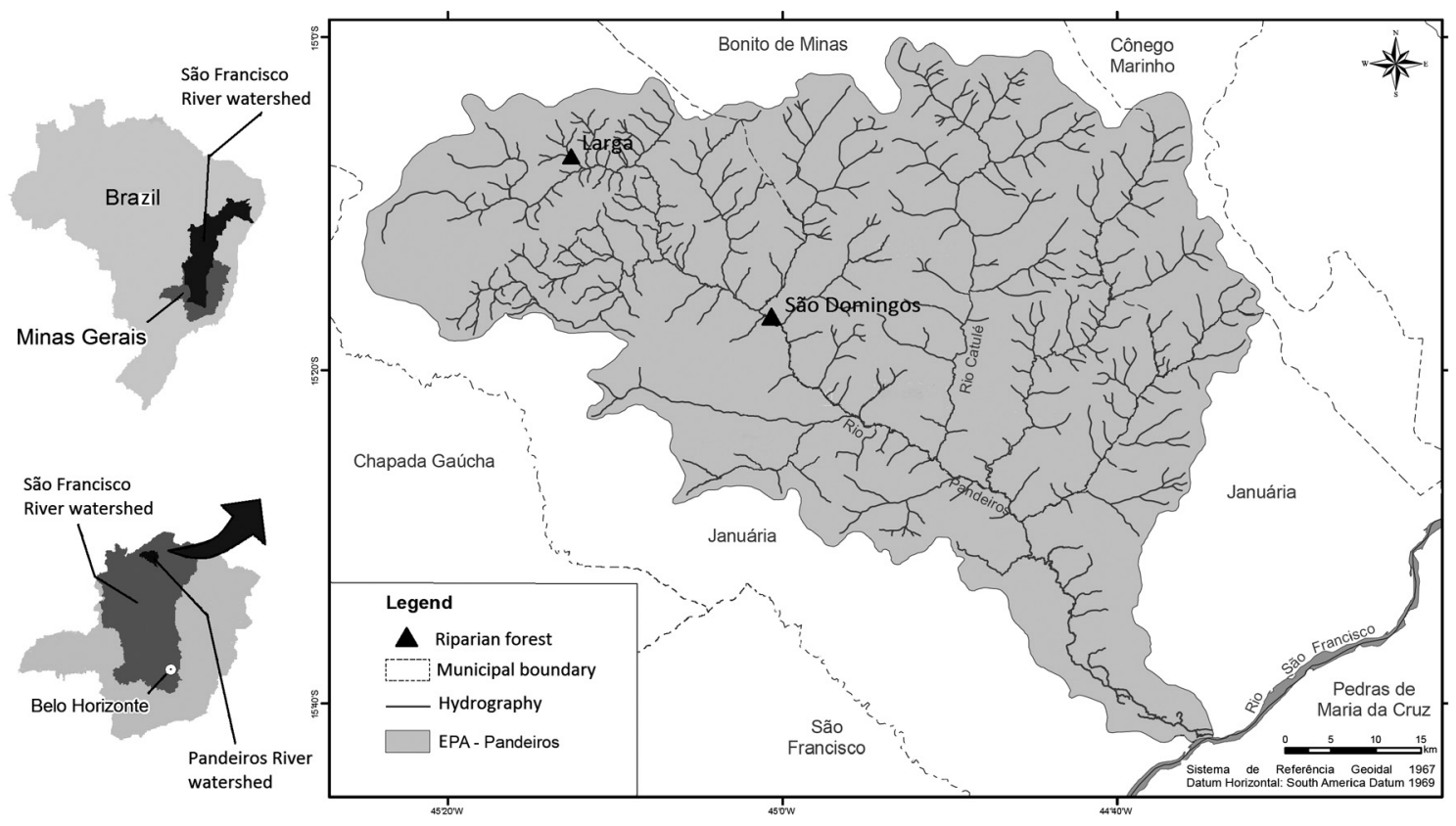

Figure 1 - Location of the stretches studied, Larga and São Domingos, in riparian vegetation of Pandeiros river, Minas Gerais, Brazil, and the Pandeiros River EPA in northern Minas Gerais, Brazil.

average temperature is around 21 to $24^{\circ} \mathrm{C}$ (Alvares et al. 2013; Azevedo et al. 2014).

The floristic and phytosociological survey was conducted in two nearby stretches along the riparian vegetation of Pandeiros river, called Larga (15 $07^{\prime} 16^{\prime \prime}$ S and $\left.45^{\circ} 12^{\prime} 22^{\prime \prime} \mathrm{W}\right)$ and São Domingos (15 $17^{\circ}$ ' $13^{\prime \prime}$ S and $\left.45^{\circ} 00^{\prime} 8^{\prime \prime} \mathrm{W}\right)$. In the first area (Larga), palm swamps are the physiognomy adjacent, and have closed riparian forest in gleysols with clay and organic character, and evidence of agriculture and livestock activities in some points (Rodrigues et al. 2009). The second area (São Domingos) is surrounded by physiognomies of Cerrado with sandy soils, features narrow riparian vegetation in some points, presence of cattle and fire evidence (Rodrigues et al. 2009).

\section{Phytosociological survey}

The survey was conducted between 2007 and 2009. We allocated 140 plots, 70 in each area (35 on the left bank and 35 on the right bank of river) of 10 $\mathrm{m} \times 10 \mathrm{~m}\left(100 \mathrm{~m}^{2}\right)$ with $10 \mathrm{~m}$ distance between plots and $3 \mathrm{~m}$ away from the flooding zone, totaling 1.4 ha of sampled area. In the plots, were inventoried all living individuals with circumference at breast height $(\mathrm{CBH}=1.30 \mathrm{~m}) \geq 15 \mathrm{~cm}$, noted the height and all of these individuals are marked with numbered aluminum tags, and collected botanical material. The botanical material was deposited in Montes
Claros Herbarium (MCMG), of the Universidade Estadual de Montes Claros (UNIMONTES). The APG IV was adopted for the classification of the species (APG IV 2016).

\section{Soil variables}

We collected five sub-samples of $500 \mathrm{~g}$ of topsoil in each plot (0-20 cm depth), and mixed to form one composite sample. The material collected was stored in plastic bags, identified and sent to Soil Analysis Laboratory, Institute of Agricultural Sciences, Universidade Federal de Minas Gerais, where it was analyzed according to the procedures of EMBRAPA (1997). The variables measured were: $\mathrm{pH}$ in water, potassium $(\mathrm{K})$, phosphorus $(\mathrm{P})$, residual phosphorus (P-res), calcium $(\mathrm{Ca})$, magnesium $(\mathrm{Mg})$, aluminum $(\mathrm{Al})$, hydrogen plus aluminum $(\mathrm{H}+\mathrm{Al})$, sum of bases $(\mathrm{SB})$, base saturation (V) effective cation exchange capacity $(\mathrm{t}), \mathrm{Al}$ saturation $(\mathrm{m})$, cation exchange capacity at $\mathrm{pH} 7.0(\mathrm{t})$, organic matter $(\mathrm{OM})$, the proportions of coarse sand (2 to $0.2 \mathrm{~mm}$ ), fine sand ( 0.2 to 0.05 $\mathrm{mm})$, silt $(0.05$ to $0.02 \mathrm{~mm})$ and clay $(<0.02 \mathrm{~mm})$.

\section{Data analysis}

The phytosociological parameters calculated were absolute (AD) and relative density (RD), absolute (AoD) and relative dominance (RoD), absolute (AF) and relative frequency (RF) and 
importance value (IV) (Mueller-Dombois \& Ellenberg 1974). To determine the alpha diversity, we calculated Shannon's diversity $\left(H^{\prime}\right)$ and Pielou's evenness $\left(J^{\prime}\right)$ (Magurran 1988). We used rarefaction method to compare species richness among sites, based on number of individuals (Gotelli \& Colwell 2001).

In order to verify differences in soil variables between sample areas, we used Analysis of Variance (ANOVA/Kruskal-Wallis) (Zar 1996), and for correlations between environmental variables and vegetation within each plot, a mixed gradient analysis was made from a Canonical Correspondence Analysis (CCA) (ter Braak 1986; ter Braak \& Prentice 1988; Palmer 1993) with matrices of species abundance and environmental variables (soil). We performed the Monte Carlo permutation test for evaluating the main axis of the significance level, and check the probabilities on the correlations between environmental variables and vegetation (ter Braak \& Smilauer 1998). All analysis was made in $\mathrm{R}$ Core Team version 3.3.1 (2016) program.

\section{Results}

Floristic and phytosociology

In total, were sampled 751 individuals, distributed in 89 species, 68 genera and 35 botanical families. The rarefaction method showed difference in richness and environmental heterogeneity between areas (Fig. 2). Larga presented 450 individuals distributed in 75 species and 31 families (Tab. 1). The diversity was 3.58 $\left(H^{\prime}\right)$, equability $0.83\left(J^{\prime}\right)$ and total basal area is $14.77 \mathrm{~m}^{2} \mathrm{ha}^{-1}$. Myracrodruon urundeuva Allemao was the most abundant species (72 individuals), and obtained higher basal area $\left(1.77 \mathrm{~m}^{2} \mathrm{ha}^{-1}\right)$ and highest values for all phytosociology parameters, consequently greater importance value (12.52\%). Siparuna guianensis Aubl. was the second species in abundance and importance value (37 individuals and $6.49 \%$, respectively), followed by Hirtella gracilipes Hook. f. (32 individuals and 4.89\%, respectively).

Tapirira obtusa (Benth.) J.D.Mitch was the second specie in basal area $\left(1.28 \mathrm{~m}^{2} \mathrm{ha}^{-1}\right)$ and



Figure 2 - Rarefaction curve of the richness estimated for Larga and São Domingos in the riparian vegetation of Pandeiros river, Minas Gerais, Brazil. 
Table 1 - Phytosociological parameters of species, ordered by the highest importance value (IV) sampled at Larga, in the riparian vegetation of Pandeiros river, Minas Gerais, Brazil. NI = number of individuals sampled; $\mathrm{BA}=$ basal area $\left(\mathrm{m}^{2} \mathrm{ha}^{-1}\right) \mathrm{RD}=$ relative density; $\mathrm{RDo}=$ relative dominance and $\mathrm{RF}=$ relative frequency.

\begin{tabular}{|c|c|c|c|c|c|c|c|}
\hline Species & Families & NI & $\mathbf{B A}$ & RD & RDo & RF & IV \\
\hline Myracrodruon urundeuva Allemão & Anacardiaceae & 72 & 1.771 & 16.00 & 12.01 & 9.58 & 12.52 \\
\hline Siparuna guianensis Aubl. & Siparunaceae & 37 & 0.532 & 8.22 & 3.60 & 7.67 & 6.49 \\
\hline Hirtella gracilipes (Hook.f.) Prance & Chrysobalanaceae & 32 & 0.598 & 7.11 & 4.05 & 3.51 & 4.89 \\
\hline Xylopia aromatica (Lam.) Mart. & Annonaceae & 22 & 0.600 & 4.89 & 4.06 & 5.43 & 4.79 \\
\hline Tapirira obtusa (Benth.) J.D.Mitch. & Anacardiaceae & 10 & 1.279 & 2.22 & 8.67 & 2.24 & 4.38 \\
\hline Acrocomia aculeata (Jacq.) Lodd. ex Mart. & Arecaceae & 12 & 0.992 & 2.67 & 6.72 & 3.19 & 4.19 \\
\hline Nectandra membranacea (Sw.) Griseb. & Lauraceae & 13 & 0.866 & 2.89 & 5.86 & 3.51 & 4.08 \\
\hline Mauritia flexuosa L.f. & Arecaceae & 7 & 0.940 & 1.56 & 6.37 & 2.24 & 3.38 \\
\hline Averrhoidium gardnerianum Baill. & Sapindaceae & 19 & 0.297 & 4.22 & 2.00 & 3.83 & 3.36 \\
\hline Cecropia pachystachya Trécul & Urticaceae & 13 & 0.505 & 2.89 & 3.42 & 2.88 & 3.06 \\
\hline Myrcia splendens (Sw.) DC. & Myrtaceae & 15 & 0.365 & 3.33 & 2.47 & 3.19 & 3.00 \\
\hline Eugenia dysenterica DC. & Myrtaceae & 15 & 0.097 & 3.33 & 0.66 & 3.51 & 2.50 \\
\hline Anadenanthera colubrina (Vell.) Brenan & Fabaceae & 7 & 0.499 & 1.56 & 3.38 & 2.24 & 2.40 \\
\hline Astronium fraxinifolium Schott ex Spreng. & Anacardiaceae & 10 & 0.114 & 2.22 & 0.77 & 2.88 & 1.96 \\
\hline Hirtella glandulosa Spreng. & Chrysobalanaceae & 8 & 0.309 & 1.78 & 2.09 & 1.92 & 1.93 \\
\hline Erythroxylum deciduum A.St.-Hil. & Erythroxylaceae & 4 & 0.529 & 0.89 & 3.58 & 1.28 & 1.91 \\
\hline Terminalia glabrescens Mart. & Combretaceae & 6 & 0.318 & 1.33 & 2.15 & 1.92 & 1.80 \\
\hline Inga laurina (Sw.) Willd. & Fabaceae & 7 & 0.365 & 1.56 & 2.47 & 1.28 & 1.76 \\
\hline Eugenia florida DC. & Myrtaceae & 9 & 0.086 & 2.00 & 0.58 & 2.55 & 1.71 \\
\hline $\begin{array}{l}\text { Coussarea hydrangeifolia (Benth.) Benth. \& Hook.f. ex } \\
\text { Müll.Arg. }\end{array}$ & Rubiaceae & 9 & 0.253 & 2.00 & 1.71 & 0.96 & 1.56 \\
\hline Byrsonima pachyphylla A. Juss. & Malpighiaceae & 8 & 0.096 & 1.78 & 0.65 & 2.24 & 1.56 \\
\hline Tapirira guianensis Aubl. & Anacardiaceae & 4 & 0.365 & 0.89 & 2.47 & 1.28 & 1.55 \\
\hline Butia capitata (Mart.) Becc. & Arecaceae & 4 & 0.409 & 0.89 & 2.77 & 0.96 & 1.54 \\
\hline Roupala montana Aubl. & Proteaceae & 7 & 0.092 & 1.56 & 0.63 & 2.24 & 1.47 \\
\hline Tachigali paniculata Aubl. & Fabaceae & 5 & 0.195 & 1.11 & 1.32 & 1.28 & 1.24 \\
\hline Diospyros hispida A.DC. & Ebenaceae & 6 & 0.069 & 1.33 & 0.47 & 1.60 & 1.14 \\
\hline Attalea oleifera Barb. Rodr. & Arecaceae & 2 & 0.299 & 0.44 & 2.03 & 0.64 & 1.03 \\
\hline Simarouba versicolor A.St.-Hil. & Simaroubaceae & 3 & 0.172 & 0.67 & 1.16 & 0.96 & 0.93 \\
\hline Ixora brevifolia Benth. & Rubiaceae & 2 & 0.264 & 0.44 & 1.79 & 0.32 & 0.85 \\
\hline Salacia elliptica (Mart. ex Schult.) G.Don & Celastraceae & 4 & 0.052 & 0.89 & 0.35 & 1.28 & 0.78 \\
\hline Tocoyena formosa (Cham. \& Schltdl.) K.Schum. & Rubiaceae & 4 & 0.026 & 0.89 & 0.18 & 1.28 & 0.75 \\
\hline Hymenaea eriogyneBenth. & Fabaceae & 4 & 0.015 & 0.89 & 0.10 & 1.28 & 0.73 \\
\hline Albizia niopoides (Spruce ex Benth.) Burkart & Fabaceae & 3 & 0.130 & 0.67 & 0.88 & 0.64 & 0.70 \\
\hline Mauritiella armata (Mart.) Burret & Arecaceae & 2 & 0.150 & 0.44 & 1.02 & 0.64 & 0.63 \\
\hline Chrysophyllum marginatum (Hook. \& Arn.) Radlk. & Sapotaceae & 3 & 0.088 & 0.67 & 0.60 & 0.64 & 0.62 \\
\hline Bauhinia longifolia (Bong.) D.Dietr. & Fabaceae & 3 & 0.085 & 0.67 & 0.58 & 0.64 & 0.62 \\
\hline Copaifera langsdorffii Desf. & Fabaceae & 2 & 0.107 & 0.44 & 0.73 & 0.64 & 0.60 \\
\hline Guettarda uruguensis Cham. \& Schltdl. & Rubiaceae & 3 & 0.026 & 0.67 & 0.18 & 0.96 & 0.60 \\
\hline Attalea speciosa Mart. ex Spreng. & Arecaceae & 2 & 0.095 & 0.44 & 0.64 & 0.64 & 0.57 \\
\hline
\end{tabular}




\begin{tabular}{|c|c|c|c|c|c|c|c|}
\hline Species & Families & NI & BA & RD & RDo & RF & IV \\
\hline Myrcia undulata O.Berg & Myrtaceae & 4 & 0.017 & 0.89 & 0.12 & 0.64 & 0.55 \\
\hline Anacardium occidentale $\mathrm{L}$. & Anacardiaceae & 2 & 0.082 & 0.44 & 0.56 & 0.64 & 0.55 \\
\hline Myrcia tomentosa (Aubl.) DC. & Myrtaceae & 3 & 0.030 & 0.67 & 0.21 & 0.64 & 0.51 \\
\hline Gochnatia polymorpha (Less.) Cabrera & Asteraceae & 3 & 0.029 & 0.67 & 0.20 & 0.64 & 0.50 \\
\hline Emmotum nitens (Benth.) Miers & Icacinaceae & 2 & 0.051 & 0.44 & 0.35 & 0.64 & 0.48 \\
\hline Piper arboreum Aubl. & Piperaceae & 2 & 0.021 & 0.44 & 0.15 & 0.64 & 0.41 \\
\hline Talisia esculenta (A.St.-Hil.) Radlk. & Sapindaceae & 2 & 0.020 & 0.44 & 0.14 & 0.64 & 0.41 \\
\hline Myrcia guianensis (Aubl.) DC. & Myrtaceae & 2 & 0.013 & 0.44 & 0.09 & 0.64 & 0.39 \\
\hline Ficus calyptroceras (Miq.) Miq. & Moraceae & 1 & 0.078 & 0.22 & 0.53 & 0.32 & 0.36 \\
\hline Terminalia januariensis DC. & Combretaceae & 3 & 0.12 & 0.67 & 0.08 & 0.32 & 0.36 \\
\hline Acosmium dasycarpum (Vogel) Yakovlev & Fabaceae & 2 & 0.025 & 0.44 & 0.18 & 0.32 & 0.31 \\
\hline Eriotheca pubescens (Mart. \& Zucc.) Schott \& Endl. & Malvaceae & 1 & 0.054 & 0.22 & 0.37 & 0.32 & 0.30 \\
\hline Magonia pubescens A.St.-Hil. & Sapindaceae & 2 & 0.008 & 0.44 & 0.06 & 0.32 & 0.27 \\
\hline Erythroxylum citrifolium A.St.-Hil. & Erythroxylaceae & 1 & 0.029 & 0.22 & 0.20 & 0.32 & 0.25 \\
\hline Ficus obtusa Hassk. & Moraceae & 1 & 0.028 & 0.22 & 0.19 & 0.32 & 0.24 \\
\hline Annona crassiflora Mart. & Annonaceae & 1 & 0.021 & 0.22 & 0.14 & 0.32 & 0.23 \\
\hline Copaifera martii Hayne & Fabaceae & 1 & 0.018 & 0.22 & 0.12 & 0.32 & 0.22 \\
\hline Pera glabrata (Schott) Poepp. ex Baill. & Euphorbiaceae & 1 & 0.017 & 0.22 & 0.12 & 0.32 & 0.22 \\
\hline Hymenaea stigonocarpa Mart. ex Hayne & Fabaceae & 1 & 0.015 & 0.22 & 0.11 & 0.32 & 0.22 \\
\hline Qualea grandiflora Mart. & Vochysiaceae & 1 & 0.015 & 0.22 & 0.11 & 0.32 & 0.22 \\
\hline Plathymenia reticulata Benth. & Fabaceae & 1 & 0.013 & 0.22 & 0.09 & 0.32 & 0.21 \\
\hline Casearia sylvestris Sw. & Salicaceae & 1 & 0.011 & 0.22 & 0.08 & 0.32 & 0.21 \\
\hline Machaerium hirtum (Vell.) Stellfeld & Fabaceae & 1 & 0.011 & 0.22 & 0.08 & 0.32 & 0.21 \\
\hline Ficus guianensis Desv. & Moraceae & 1 & 0.009 & 0.22 & 0.06 & 0.32 & 0.20 \\
\hline Inga vera Willd. & Fabaceae & 1 & 0.009 & 0.22 & 0.06 & 0.32 & 0.20 \\
\hline Casearia ulmifolia Vahl & Salicaceae & 1 & 0.009 & 0.22 & 0.06 & 0.32 & 0.20 \\
\hline Curatella americana $\mathrm{L}$. & Dilleniaceae & 1 & 0.008 & 0.22 & 0.06 & 0.32 & 0.20 \\
\hline Myrsine umbellata Mart. & Primulaceae & 1 & 0.006 & 0.22 & 0.04 & 0.32 & 0.20 \\
\hline Chomelia sericea Müll. Arg. & Rubiaceae & 1 & 0.006 & 0.22 & 0.04 & 0.32 & 0.19 \\
\hline Miconia albicans (Sw.) Triana & Melastomataceae & 1 & 0.005 & 0.22 & 0.04 & 0.32 & 0.19 \\
\hline Bauhinia acuruana Moric. & Fabaceae & 1 & 0.005 & 0.22 & 0.04 & 0.32 & 0.19 \\
\hline Vernonanthura phosphorica (Vell.) H.Rob. & Asteraceae & 1 & 0.004 & 0.22 & 0.03 & 0.32 & 0.19 \\
\hline Ficus umbellata Vahl & Moraceae & 1 & 0.004 & 0.22 & 0.03 & 0.32 & 0.19 \\
\hline Ouratea castaneifolia (DC.) Engl. & Ochnaceae & 1 & 0.004 & 0.22 & 0.03 & 0.32 & 0.19 \\
\hline Matayba guianensis Aubl. & Sapindaceae & 1 & 0.004 & 0.22 & 0.03 & 0.32 & 0.19 \\
\hline Cordiera concolor (Cham.) Kuntze & Rubiaceae & 1 & 0.003 & 0.22 & 0.02 & 0.32 & 0.19 \\
\hline
\end{tabular}

relative dominance $(8.67 \%)$, followed by the palms Acrocomia aculeata (Jacq.) Lodd. ex Mart. (BA = $0.99 \mathrm{~m}^{2} \mathrm{ha}^{-1}$ and $6.72 \%$ of relative dominance) and Mauritia flexuosa L.f. (BA $=0.94 \mathrm{~m}^{2} \mathrm{ha}^{-1}$ and $6.37 \%$ of relative dominance). The richest families were Fabaceae (14 species), Arecaceae, Myrtaceae and
Rubiaceae (six species each), Anacardiaceae (five species), Moraceae and Sapindaceae (four species each), Annonaceae, Asteraceae, Chrysobalanaceae, Combretaceae, Erythroxylaceae and Salicaceae (two species each). Eighteen families were represented by only one individual. 
São Domingos were 301 individuals, distributed in 40 species and 24 families (Tab. 2). The diversity, equability and total basal area were respectively $2.45,0.66$ and $3.82 \mathrm{~m}^{2} \mathrm{ha}^{-1}$, lower values than that found in Larga, including richness and abundance. The most abundant specie was Curatella americana L. (132 individuals; importance value of $35.64 \%$ ), which obtained the highest values for all phytosociological parameters, and represented $44 \%$ of all individuals sampled in this area. The second species in abundance (22 individuals), importance value $(6.66 \%)$ and relative frequency $(9.14 \%)$ was Erythroxylum deciduum A. St.-Hill.

Mauritia flexuosa was the species with the second largest basal area $\left(0.63 \mathrm{~m}^{2} \mathrm{ha}^{-1}\right)$ and relative dominance (16.61\%), followed by Xylopia aromatica (Lam.) Mart., with $0.24 \mathrm{~m}^{2} \mathrm{ha}^{-1}$ of basal area and $6.41 \%$ of dominance. Families who have greater number of species were Fabaceae (seven species), Anacardiaceae (four species), Arecaceae and Myrsinaceae (three species each), and eighteen families contributed with only species each.

Table 2 - Phytosociological parameters of species, ordered by highest importance value (IV) sampled at São Domingos, in the riparian vegetation of Pandeiros river, Minas Gerais, Brazil. NI = number of individuals sampled; BA $=$ basal area $\left(\mathrm{m}^{2} \mathrm{ha}^{-1}\right) \mathrm{RD}=$ relative density; $\mathrm{RDo}=$ relative dominance and $\mathrm{RF}=$ relative frequency.

\begin{tabular}{|c|c|c|c|c|c|c|c|}
\hline Species & Families & NI & BA & RD & RDo & RF & IV \\
\hline Curatella americana $\mathrm{L}$. & Dilleniaceae & 132 & 1.339 & 43.85 & 35.07 & 28.00 & 35.64 \\
\hline Erythroxylum deciduum A.St.-Hil. & Erythroxylaceae & 22 & 0.135 & 7.31 & 3.54 & 9.14 & 6.66 \\
\hline Mauritia flexuosa L.f. & Arecaceae & 4 & 0.634 & 1.33 & 16.61 & 1.71 & 6.55 \\
\hline Xylopia aromatica (Lam.) Mart. & Annonaceae & 14 & 0.245 & 4.65 & 6.41 & 6.29 & 5.78 \\
\hline Tapirira guianensis Aubl. & Anacardiaceae & 15 & 0.112 & 4.98 & 2.92 & 5.71 & 4.54 \\
\hline Hirtella gracilipes (Hook.f.) Prance & Chrysobalanaceae & 13 & 0.124 & 4.32 & 3.24 & 4.57 & 4.04 \\
\hline Myrcia tomentosa (Aubl.) DC. & Myrtaceae & 13 & 0.066 & 4.32 & 1.73 & 4.57 & 3.54 \\
\hline Averrhoidium garnerianum Baill. & Sapindaceae & 14 & 0.069 & 4.65 & 1.82 & 2.86 & 3.11 \\
\hline Hirtella glandulosa Spreng. & Chrysobalanaceae & 8 & 0.124 & 2.66 & 3.24 & 2.86 & 2.92 \\
\hline Copaifera langsdorffii Desf. & Fabaceae & 5 & 0.085 & 1.66 & 2.22 & 2.29 & 2.06 \\
\hline Byrsonima pachyphylla A.Juss. & Malpighiaceae & 6 & 0.037 & 1.99 & 0.97 & 2.86 & 1.94 \\
\hline Mauritiella armata (Mart.) Burret & Arecaceae & 1 & 0.168 & 0.33 & 4.40 & 0.57 & 1.77 \\
\hline Cecropia pachystachya Trécul & Cecropiaceae & 5 & 0.024 & 1.66 & 0.63 & 2.86 & 1.72 \\
\hline Myrsine coriacea (Sw.) Roem. \& Schult. & Primulaceae & 5 & 0.038 & 1.66 & 1.00 & 2.29 & 1.65 \\
\hline Buchenavia tomentosa Eichler & Combretaceae & 3 & 0.103 & 1.00 & 2.71 & 1.14 & 1.62 \\
\hline Diospyros sericea A.DC. & Ebenaceae & 2 & 0.113 & 0.66 & 2.96 & 1.14 & 1.59 \\
\hline Astronium fraxinifolium Schott ex Spreng. & Anacardiaceae & 4 & 0.014 & 1.33 & 0.36 & 2.29 & 1.33 \\
\hline Myrcia splendens (Sw.) DC. & Myrtaceae & 4 & 0.028 & 1.33 & 0.75 & 1.71 & 1.26 \\
\hline Acrocomia aculeata (Jacq.) Lodd. ex Mart. & Arecaceae & 1 & 0.098 & 0.33 & 2.57 & 0.57 & 1.16 \\
\hline Baccharis serrulata Pers. & Asteraceae & 3 & 0.014 & 1.00 & 0.37 & 1.71 & 1.03 \\
\hline Simarouba versicolor A.St.-Hil. & Simaroubaceae & 2 & 0.040 & 0.66 & 1.05 & 1.14 & 0.95 \\
\hline $\begin{array}{l}\text { Tabebuia aurea (Manso) Benth. \& Hook.f. ex } \\
\text { S.Moore }\end{array}$ & Bignoniaceae & 1 & 0.065 & 0.33 & 1.72 & 0.57 & 0.87 \\
\hline Kielmeyera coriaceae Mart. \& Zucc. & Clusiaceae & 2 & 0.149 & 0.66 & 0.39 & 1.14 & 0.73 \\
\hline Eugenia dysenterica DC. & Myrtaceae & 2 & 0.014 & 0.66 & 0.37 & 1.14 & 0.73 \\
\hline Tachigali paniculata Aubl. & Fabaceae & 2 & 0.013 & 0.66 & 0.34 & 1.14 & 0.72 \\
\hline Myrsine umbellata Mart. & Primulaceae & 2 & 0.008 & 0.66 & 0.23 & 1.14 & 0.68 \\
\hline Myrsine guianensis (Aubl.) Kuntze & Primulaceae & 2 & 0.006 & 0.66 & 0.15 & 1.14 & 0.65 \\
\hline Euplassa rufa (Loes.) Sleumer & Proteaceae & 2 & 0.010 & 0.66 & 0.26 & 0.57 & 0.50 \\
\hline Tocoyena formosa (Cham. \& Schltdl.) K. Schum. & Rubiaceae & 1 & 0.013 & 0.33 & 0.35 & 0.57 & 0.42 \\
\hline
\end{tabular}




\begin{tabular}{lllllllc}
\hline \multicolumn{1}{c}{ Species } & \multicolumn{1}{c}{ Families } & NI & BA & RD & RDo & RF & IV \\
\hline Inga laurina (Sw.) Willd. & Fabaceae & 1 & 0.012 & 0.33 & 0.33 & 0.57 & 0.41 \\
Myracrodruon urundeuva Allemão & Anacardiaceae & 1 & 0.010 & 0.33 & 0.25 & 0.57 & 0.38 \\
Dimorphandra mollis Benth. & Fabaceae & 1 & 0.009 & 0.33 & 0.24 & 0.57 & 0.38 \\
Acosmium dasycarpum (Vogel) Yakovlev & Fabaceae & 1 & 0.006 & 0.33 & 0.16 & 0.57 & 0.35 \\
Macairea radula (Bonpl.) DC. & Melastomataceae & 1 & 0.006 & 0.33 & 0.15 & 0.57 & 0.35 \\
Ouratea castaneifolia (DC.) Engl. & Ochnaceae & 1 & 0.004 & 0.33 & 0.12 & 0.57 & 0.34 \\
Gochnatia polymorpha (Less.) Cabrera & Asteraceae & 1 & 0.003 & 0.33 & 0.10 & 0.57 & 0.33 \\
Lafoensia pacari A.St.-Hil. & Lythraceae & 1 & 0.003 & 0.33 & 0.10 & 0.57 & 0.33 \\
Andira anthelmia (Vell.) Benth. & Fabaceae & 1 & 0.003 & 0.33 & 0.08 & 0.57 & 0.33 \\
Bowdichia virgilioides Kunth & Fabaceae & 1 & 0.002 & 0.33 & 0.07 & 0.57 & 0.32 \\
Mangifera indica L. & Anacardiaceae & 1 & 0.002 & 0.33 & 0.07 & 0.57 & 0.32 \\
\hline
\end{tabular}

\section{Soil characteristics}

Most soil properties showed different levels among sampled areas (Tab. 3). However, magnesium, aluminum, aluminum saturation and base saturation showed similar levels. In São Domingo's soil, the attributes $\mathrm{pH}$ in water, fine sand, phosphorus and residual phosphorus obtained the highest values, showing a sandy soil.

Table 3 - Comparison between soil attributes of Larga and São Domingos in the riparian vegetation of Pandeiros river, Minas Gerais, Brazil: $\mathrm{pH}$ in water $(\mathrm{pH})$, potassium $(\mathrm{K})$, phosphorus (P- Mehlich), residual phosphorus (P- rem), potassium $\left(\mathrm{K}^{+}\right)$, calcium $(\mathrm{Ca})$, magnesium $(\mathrm{Mg})$, aluminum $(\mathrm{Al})$, hydrogen plus aluminum $(\mathrm{H}+\mathrm{Al})$, total bases $(\mathrm{SB})$, effective cation exchange capacity at $\mathrm{pH} 7.0(\mathrm{t})$, aluminum saturation $(\mathrm{m})$, cation exchange capacity $(\mathrm{T})$, base saturation $(\mathrm{V})$, organic matter $(\mathrm{OM})$, coarse sand proportions ( 2 to $0.2 \mathrm{~mm})$, fine sand $(0.2$ to $0.05 \mathrm{~mm})$, silt (0.05 to $0.02 \mathrm{~mm})$ and clay $(<0.02 \mathrm{~mm})$. * Indicates normal distribution of data.

\begin{tabular}{|c|c|c|c|c|c|}
\hline \multirow[b]{2}{*}{ Soil variables } & \multirow[b]{2}{*}{ Larga } & \multirow[b]{2}{*}{ São Domingos } & \multicolumn{3}{|c|}{ ANOVA/Kruskal-Wallis } \\
\hline & & & $F / X^{2}{ }_{(1,126)}$ & $\mathbf{P}$ & \\
\hline $\mathrm{pH}$ & $5.84 \pm 0.06$ & $6.12 \pm 0.04$ & 9.084 & 0.0026 & \\
\hline P-Mehlich (mg.kg-1) & $1.68 \pm 0.31$ & $1.69 \pm 0.16$ & 4.541 & 0.0331 & \\
\hline P-rem (mg.L-1) & $50.51 \pm 0.33$ & $52.62 \pm 0.38$ & 13.111 & 0.0003 & \\
\hline $\mathrm{K}^{+}\left(\mathrm{cmolc} \cdot \mathrm{dm}^{-3}\right)$ & $33.43 \pm 1.48$ & $26.42 \pm 1.62$ & 10.272 & 0.0017 & $*$ \\
\hline $\mathrm{Ca}^{++}\left(\mathrm{cmolc} \cdot \mathrm{dm}^{-3}\right)$ & $1.49 \pm 0.08$ & $1.29 \pm 0.05$ & 3.894 & 0.0485 & \\
\hline $\mathrm{Mg}^{++}\left(\mathrm{cmolc} \cdot \mathrm{dm}^{-3}\right)$ & $0.6 \pm 0.03$ & $0.57 \pm 0.03$ & 0.159 & 0.6902 & \\
\hline $\mathrm{Al}\left(\mathrm{cmolc} \cdot \mathrm{dm}^{-3}\right)$ & $0.06 \pm 0.02$ & $0.03 \pm 0.01$ & 0.027 & 0.8697 & \\
\hline $\mathrm{H}+\mathrm{Al}\left(\mathrm{cmolc}^{\left.-\mathrm{dm}^{-3}\right)}\right.$ & $1.51 \pm 0.06$ & $1.21 \pm 0.04$ & 13.349 & 0.0003 & \\
\hline $\mathrm{SB}\left(\mathrm{cmolc} \cdot \mathrm{dm}^{-3}\right)$ & $2.18 \pm 0.1$ & $1.93 \pm 0.08$ & 3.774 & 0.0543 & $*$ \\
\hline $\mathrm{t}\left(\mathrm{cmolc} \cdot \mathrm{dm}^{-3}\right)$ & $2.24 \pm 0.1$ & $1.96 \pm 0.07$ & 5.322 & 0.0227 & $*$ \\
\hline m (\%) & $3.92 \pm 1.19$ & $2.01 \pm 0.59$ & 0.027 & 0.8697 & \\
\hline $\mathrm{T}\left(\mathrm{cmolc} \cdot \mathrm{dm}^{-3}\right)$ & $3.69 \pm 0.11$ & $3.14 \pm 0.08$ & 16.168 & 0.0001 & $*$ \\
\hline V $(\%)$ & $58.44 \pm 1.74$ & $60.63 \pm 1.27$ & 0.794 & 0.3728 & \\
\hline $\mathrm{OM}\left(\right.$ dag. $\left.\mathrm{kg}^{-1}\right)$ & $2.27 \pm 0.07$ & $1.68 \pm 0.05$ & 30.914 & $<0.0001$ & \\
\hline Coarse sand (dag.kg-1 $)$ & $27.2 \pm 1.52$ & $10.45 \pm 1.43$ & 43.504 & $<0.0001$ & \\
\hline Fine sand (dag.kg-1 $\left.{ }^{-1}\right)$ & $60.34 \pm 1.58$ & $84.01 \pm 1.54$ & 115.129 & $<0.0001$ & * \\
\hline Silt (dag. $\left.\mathrm{kg}^{-1}\right)$ & $5.6 \pm 0.31$ & $2.95 \pm 0.15$ & 33.053 & $<0.0001$ & \\
\hline Clay (dag.kg-1 $\left.{ }^{-1}\right)$ & $6.28 \pm 0.21$ & $3.87 \pm 0.14$ & 61.929 & $<0.0001$ & \\
\hline
\end{tabular}


While in Larga, potassium, calcium, hydrogen plus aluminum, cation exchange capacity at $\mathrm{pH} 7.0$, organic matter, the proportions of coarse sand, silt and clay had the highest values, showing that the soil in this area is more clayey and rich in organic matter.

The CCA showed segregation between areas, with formation of three groups: two comprising the plots located in Larga (most plots are correlated with clay and organic matter, and some plots are correlated with aluminum content), and one comprising plots of São Domingos (Fig. 3a). The distribution of species occurs according the soil properties of each area (Fig. 3b), where species recognized as characteristics of peat swamps occurs in Larga, in most clay and swampy soils. At the same way, the species more related with sandy soils occurs in São Domingos area, stretch that has acid soils such as those found in Cerrado areas.

The eigenvalue of first axis of CCA $(0.55$, Fig. 3b) was considered high ( $>0.5$ by ter Braak 1995), indicating the existence of a gradient with species substitution between the two extremes, and moderate for axis $2(0.41)$, assuming that the gradients indicated by the analysis are long for axis 1 and relatively short for axis 2 , with the distribution of the species over the extremes. The two axis explained a cumulative of $8.2 \%$ of the variance of the data, indicating that the remaining variance unexplained is great. On other hand, the species-environment correlations were high (Tab. 4), and Monte Carlo test showed that the abundance of species was significantly correlated with soil properties $(\mathrm{p}<0.01)$. The environmental variables positively correlated with axis 1 were base saturation and fine sand, and negatively are aluminium, aluminum saturation, organic matter, coarse sand and clay. The axis 2 was positively correlated with base saturation, organic matter, coarse sand, fine sand and clay, and negatively with aluminum and aluminum saturation (Tab. 5).

\section{Discussion}

The diversity and evenness values found in this study reflected the transition area of Pandeiros river, with several phytophysiognomies types of Cerrado and Caatinga domains (Azevedo et al. 2014; Veloso et al. 2014; Ávila et al. 2016). Riparian forests connect different types of vegetation and adjacent flora, and soil and hydromorphic conditions create a spatial heterogeneity that favors species richness in these environments (Veloso et al. 2014; Corenblit et al. 2015).

The structural parameters, diversity and evenness varied among the sampled areas, as hypothesized. Larga showed higher values for structure parameters, explained by low density and dominance of species sampled. In turn, São
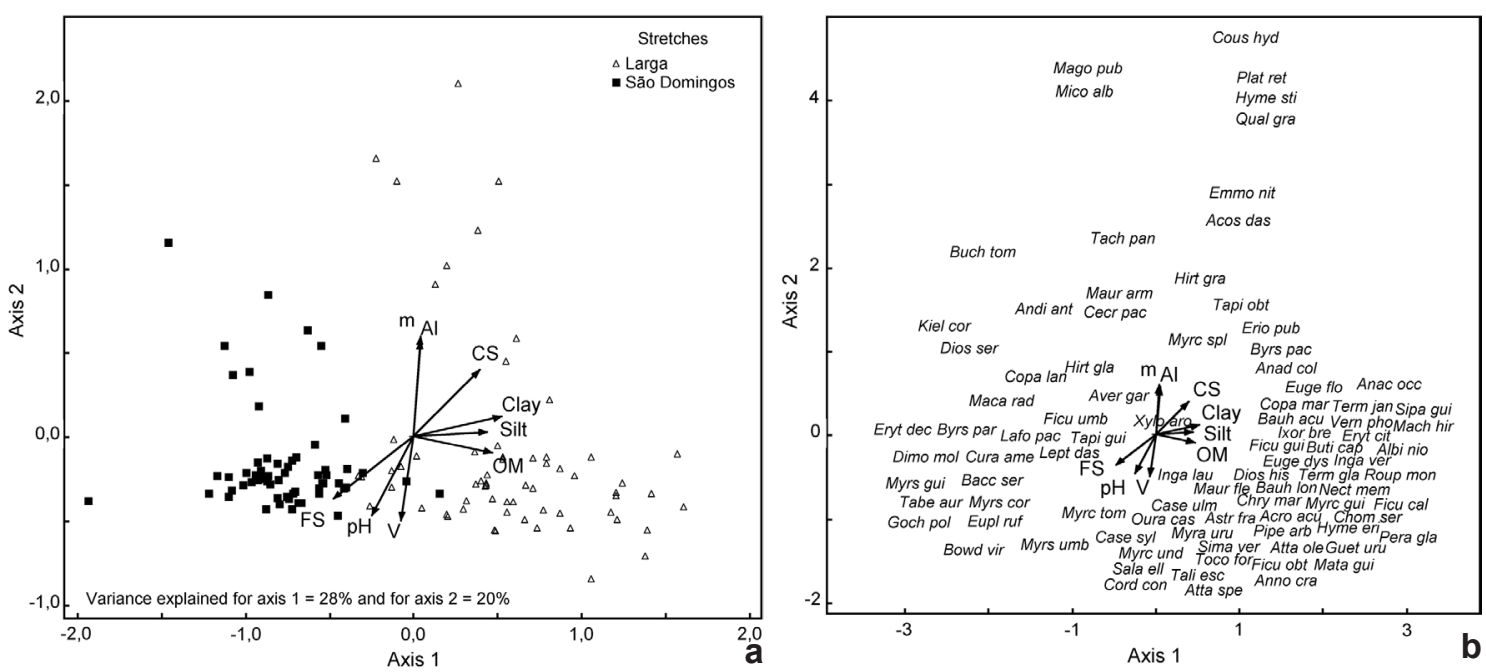

Figure 3 - Diagram of Canonical Correspondence Analysis (CCA), with the distribution of plots (a) and species (b) in Larga and São Domingos stretches, in the riparian vegetation of Pandeiros river, Minas Gerais, Brazil, and their correlation with environmental variables: $\mathrm{pH}$ in water $(\mathrm{pH})$, aluminum (Al), aluminum saturation (m), base saturation $(\mathrm{V})$, organic matter (OM), fine sand (FS), coarse sand (CS), silt and clay. 
Table 4 - Summary presenting the eigenvalues for the first three axis, percentage of variance explained by each axis and species-environment correlation coefficients sampled at Larga and São Domingos, in the riparian vegetation of Pandeiros river, Minas Gerais, Brazil. Total variance (or inertia) on the data of species: 18:52.

\begin{tabular}{lccc}
\hline Summary & Axis1 & Axis2 & Axis3 \\
\hline Eigen value & 0.55 & 0.41 & 0.31 \\
Variance on the species data & & & \\
\% of explained variance & 3 & 2.2 & 1.6 \\
\% of cumulative variance & 3 & 5.2 & 6.8 \\
Pearson's correlation, species-environment & 0.88 & 0.88 & 0.82 \\
Kendall's correlation (Rank), species-environment & 0.59 & 0.42 & 0.45 \\
\hline
\end{tabular}

Table 5 - Canonical Correspondence Analysis (CCA) of abundance of 89 species sampled at Larga and São Domingos, in the riparian vegetation of Pandeiros river, Minas Gerais, Brazil. The values represent the intraset between environmental variables and the first two axis of ordination, and the correlation matrix weighted between environmental variables: $\mathrm{pH}$ in water $(\mathrm{pH})$, aluminum $(\mathrm{Al})$, aluminum saturation $(\mathrm{m})$, base saturation $(\mathrm{V})$, organic matter (OM), fine sand (FS), coarse sand (CS), silt and clay.

\begin{tabular}{lcccccccccc}
\hline \multirow{2}{*}{ Soil variables } & \multicolumn{2}{c}{ intraset } & \multicolumn{9}{c}{ Soil variables } \\
& Axis1 & Axis2 & pH & Al & m & V & O.M & F.S & C.S & Silt \\
\hline $\mathrm{pH}$ & 0.34 & 0.58 & & & & & & & & \\
$\mathrm{Al}$ & -0.09 & -0.75 & -0.82 & & & & & & & \\
$\mathrm{~m}$ & -0.05 & -0.61 & -0.82 & 0.95 & & & & & & \\
V & 0.14 & 0.65 & 0.83 & -0.87 & -0.87 & & & & \\
M.O & -0.55 & 0.05 & 0.04 & -0.19 & -0.24 & 0.24 & & & \\
A.G & -0.62 & -0.29 & -0.55 & 0.45 & 0.41 & -0.47 & 0.15 & & & \\
A.F & 0.53 & 0.55 & 0.66 & -0.65 & -0.54 & 0.62 & -0.09 & -0.84 & & \\
Silt & -0.57 & -0.02 & -0.35 & 0.22 & 0.19 & -0.20 & 0.35 & 0.34 & -0.40 & \\
Clay & -0.60 & -0.08 & -0.41 & 0.21 & 0.16 & -0.24 & 0.37 & 0.37 & -0.42 & 0.16 \\
\hline
\end{tabular}

Domingos showed high density of one species and low basal area, which is a typical dominance pattern presented by forest formations under unfavorable environmental conditions such as shallow or nutrient-poor soils (Souza et al. 2007). The fertility and physical characteristics is an environmental filter and can be a limiting factor for species richness (Lemos et al. 2013). Thus, the environmental heterogeneity observed in Larga and São Domingos soils confers the patterns of floristic variation between these areas. The lower values of diversity and basal area presented by São Domingos may also be related to anthropic pressure existing in this area, with selective cut of arboreal species and frequent fire occurrence.

Curatella americana, species dominant in São Domingos area, is a pioneer and generalist species characteristic of savanna, and its high abundance was influenced by cerrado vegetation in this area (Rodrigues et al. 2009). Likewise, Erythroxylum deciduum is a pioneer species, selective hygrophytes, and with wide distribution in several forest formations including gallery forests and cerrado (Lorenzi 1992). The high importance value of pioneer species in São Domingos reinforces the limiting character of the habitat, were generalist species being favored. The anthropic activities in this study area also be contributed with this high dominance of generalists.

Larga area features typical vegetation of riparian forest, with larger trees and more humid environment, even in plots with more aluminum content. According to Dantas \& Batalha (2011), in cerrado areas, the plant available water 
together with soil characteristics could explain the vegetation distribution at local scales. In this area, Myracrodruon urundeuva is the most important tree, indicator of fertility soil (Souza et al. 2007) and typical deciduous forest species, appears on the brazilian's official list threatened flora, in the vulnerable class (Arruda et al. 2013; Martinelli \& Moraes 2013). Siparuna guianensis and Hirtella gracilipes also showed high abundance in Larga. These species are typical of riparian vegetation (Rodrigues et al. 2009). The presence of cerrado, dry and moist forest's species in studied areas highlights the influence of these vegetation formations in floristic composition of Pandeiros river riparian vegetation and expresses ecotonal character of the northern region of Minas Gerais (Arruda et al. 2013). The influence of adjacent vegetation types in riparian vegetation was also recorded in other floristic and structural studies in the region (Santos \& Vieira 2006; Menino et al. 2009; Rodrigues et al. 2009; Sales et al. 2009; Menino et al. 2012; Veloso et al. 2014).

Corroborating hypothesis, soil variables differed between areas. Larga presented fertile soils, clayey and rich in organic matter with higher cation exchange capacity and slightly acidic, mainly due to the presence of potassium and hydrogen ions (related probably to higher nutrient cycling), and São Domingos presents sandy soils. The proximity with palm swamps can be defined gleysols of Larga and anaerobic conditions imposed on clay soil by flooding contribute to the release of aluminum, causing higher aluminum saturation $(\mathrm{Al}+\mathrm{H})$ in some plots (Ronquim 2010). Levels between 50-80\% of total base saturation characterized fertile soils (Ronquim 2010), such as soils found in Larga and São Domingos. However, São Domingos presents more sandy soils, with high phosphorus content, low organic matter and fire evidence in some points. The practice of burning may increase the present level of phosphorus in soil, mainly due to incorporation of ashes, but in long term can cause physical and chemical degradation of soil (Ketterings et al. 2002).

In both areas, soils played a key role in composition and structure of community. The soils rich in organic matter, as found in Larga, is related to high soil fertility and allows water and air to move through the soil, facilitating roots penetration and providing clay aggregation stability and nutrients for plants (Dantas \& Batalha 2011). Thus, fertile soils tends to present higher richness by allows to coexistence of more species and higher basal area by allows enhanced of biomass gain (Souza et al. 2007; Dantas \& Batalha 2011). Most of Larga species was related to clay and organic soil character, probably because gleysol favors hygrophilous species.

Some of species, like Inga laurina (Sw.) Willd, Byrsonima pachyphylla A. Juss., Cecropia pachystachya, Hirtella gracilipes, Myrcia splendens (Sw) DC, Myrsine umbellata Mart., Nectandra membranaceae (Sw) Griseb. e Tapirira guianensis Aubl was described by Ávila et al. (2016) in a palm swamp in EPA Pandeiros River, near to our study area, and occurred in both areas sampled. However, Hirtella gracilipes, Myrcia splendens and Cecropia pachystachya are related of aluminum and aluminum saturation, commonly abundant in cerrado soils, which may denote a generalist character of these species. Mauritia flexuosa is described as typical specie of palm swamps (Fagundes \& Ferreira 2016), and it occurred in both areas to. Typical species of dry forests, like Myracrodruon urundeuva and Talisia esculenta (A.St.) Radlk have higher affinity for soils rich in organic matter (Madeira et al. 2009; Arruda et al. 2013; Reis et al. 2016), and showed a greater association with Larga soils. Myracrodruon urundeuva is cited by Rossato et al. (2015) as specie largely associated with soils rich in macronutrients, especially $\mathrm{Ca}$ and $\mathrm{Mg}$, presents in soils of dry forests. On other hand, Larga present plots on acid soils with higher soil aluminum content and lower organic matter, typical of Cerrado (Haridasan 2008), where predominate cerrado's species like Xylopia aromatica (Lam.) Mart and Hymenaea stigonocarpa Mart. ex Hayne.

Despite the proximity between areas, Larga and São Domingos presents differences in soils conditions, that's influences structure and composition. Riparian forests support high biodiversity and are important in maintenance of riverine ecosystem (Azevedo et al. 2014). Even though areas are preserved by law in Brazil, anthropogenic impacts as fire or selective cutting can modify these environments (Ketterings et al. 2002; Azevedo et al. 2014). When riparian forests occurs in ecotonal areas, as in study areas, presents a peculiarities in composition that's justified more attention in management and conservation of this environments. 


\section{Conclusions}

Overall, the present study indicate that soil attributes can influence the patterns of richness, structure, dominance and the establishment of different species, and the composition is also influenced by ecotonal character. The soils fertility and physical characteristics in these study areas can be a limiting factor that acts as an environmental filter for establishment of species. Larga presents higher richness, evenness, basal area than São Domingos area, and species associated with mesotrophic soils. Thus, in ecotonal areas of Cerrado / Caatinga, the increased of soil fertility allows a higher productivity, coexistence of more species and a replaced of species characteristic of cerrado by forest species. Despite of low distance between the sampled areas, these are singular environments influenced by different soils, by the mixed composition of the ecotonal area and the anthropogenic impacts to which they are exposed.

\section{Acknowledgements}

We thanks to Conselho Nacional de Pesquisa Científica - CNPq (555980/2006-5) for funded this study, FAPEMIG (MDM Veloso, BIP 0186-15, PPM 0623-16; YRF Nunes PPM 627-16; and Phd scholarship of MA Ávila), CAPES (Phd scholarship of NCA Fagundes) and CNPq (YRF Nunes, 306375/2016-8) for the support provide to authors. We also thank the students of the Laboratório de Ecologia Vegetal of Universidade Estadual de Montes Claros for their help with fieldwork, and the Instituto Estadual de Floresta (IEF/MG) for logistic support.

\section{References}

Alvares CA, Stape JL, Sentelhas PC, Gonçalves JLM \& Sparovek G (2013) Koppen's climate classification map for Brazil. Meteorologische Zeitschrift 22: 711-728.

APG IV (2016) An update of Angiosperm Plylogeny Group classification for the orders and families of flowering plants: APG IV. Botanical Journal of the Linnean Society 181: 1-20.

Arruda DM, Ferreira-Júnior WG, Duque-Brasil R \& Schaefer CER (2013) Phytogeographical patterns of dry forests sensu stricto in northern Minas Gerais State, Brazil. Anais Academia Brasileira de Ciências 85: 283-294.

Azevedo IFP, Nunes YRF, Ávila MA, Silva DL, Fernandes GW \& Veloso RB (2014) Phenology of riparian tree species in a transitional region in southeastern Brazil. Brazilian Journal of Botany 37: 47-59.
Bennett AF, Nimmo DG \& Radford JQ (2014) Riparian vegetation has disproportionate benefits for landscape-scale conservation of woodland birds in highly modified environments. Journal of Applied Ecology 51: 514-523.

Caiafa AN \& Martins FR(2007) Taxonomic identification, sampling methods, and minimum size of the tree sampled: implications and perspectives for studies in the Brazilian Atlantic Rainforest. Functional Ecosystems and Communities 1: 95-104.

Corenblit D, Davies NS, Steiger J, Gibling MR \& Bornette G (2015) Considering river structure and stability in the light of evolution: feedbacks between riparian vegetation and hydrogeomorphology. Earth Surface Processes and Landforms 40: 189-207.

Dantas VL \& Batalha MA (2011) Vegetation structure: fine scale relationships with soil in a cerrado site. Flora 206: 341-346.

Dietrich AL, Nilsson C \& Jansson R (2015) Restoration effects on germination and survival of plants in the riparian zone: a phytometer study. Plant Ecology 216: 465-477.

Embrapa - Empresa Brasileira de Agropecuária (1997) Manual de métodos de análise de solo. Embrapa Solos, Rio de Janeiro. 212p.

Embrapa - Empresa Brasileira de Agropecuária (2013) Sistema brasileiro de classificação de solos. $3^{\text {a }}$ ed. Embrapa, Brasília. 353p.

Gotelli NJ \& Colwell AM (2001) Quantifying biodiversity: procedures and pitfalls in the measurement and comparison of species richness. Ecology Letters 4: 379-391.

Ketterings QM, Noordwijk MV \& Bigham JM (2002) Soil phosphorus availability after slash-and-burn fires of different intensities in rubber agroforests in Sumatra, Indonesia. Agriculture, Ecosystems and Environment 92: 37-48.

Lemos HL, Pinto JRR, Mews HA \& Lenza E (2013) Structure and floristic relationships between Cerrado sensu stricto sites on two types of substrate in northern Cerrado, Brazil. Biota Neotropica 4: 121-132.

Lloyd J, Domingues TF, Schrodt F, Ishida FY, Feldpausch TR, Saiz G, Quesada CA, Schwarz M, TorelloRaventos M, Gilpin M, Marimon BS, MarimonJunior BH, Ratter JA, Grace J, Nardoto GB, Veenendaal E, Arroyo L, Villarroel D, Killeen TJ, Steiningera M \& Phillips OL (2015) Edaphic, structural and physiological contrasts across Amazon Basin forest-savanna ecotones suggest a role for potassium as a key modulator of tropical woody vegetation structure and function. Biogeosciences 12: 6529-6571.

Lorenzi H (1992) Árvores brasileiras: manual de identificação e cultivo de plantas arbóreas nativas do Brasil. Plantarum, Nova Odessa. 352p. 
Madeira BG, Espírito-Santo MM, D’ângelo-Neto S, Nunes YRF, Sanchez-Azofeifa GA, Fernandes GW \& Quesada M (2009) Changes in tree and liana communities along a successional gradient in a tropical dry forest in south-eastern Brazil. Plant Ecology 201: 291-304.

Magurran AE (1988) Ecological diversity and its measurement. Princeton University Press, Princeton. $192 p$.

Mueller-Dombois D \& Ellenberg H (1974) Aims and methods of vegetation ecology. John Wiley \& Sons, New York. 574p.

Palmer MW (1993) Putting things in even better order: the advantages of canonical correspondence analysis. Ecology 74: 2215-2230.

R Core Team (2016) Version 3.3.1 "Bug in Your Hair". $\mathrm{R}$ : A language and environment for statistical computing. R Foundation for Statistical Computing, Vienna. Available at $<$ https://www.R-project.org/> . Access on September 2017.

Rodrigues PMS, Azevedo IFP, Veloso MDM, Santos RM, Menino GCO, Nunes YRF \& Fernandes GW (2009) Diversidade florística da vegetação ciliar do rio Pandeiros, norte de Minas Gerais. MG Biota 2: $18-35$.

Ronquim CC (2010) Conceitos de fertilidade do solo e manejo adequado para as regiões tropicais. Boletim de pesquisa e desenvolvimento. Embrapa Monitoramento por Satélite, Campinas. 26p.

Rossi M, Mattos IFA, Coelho RM, Menk JRF, Rocha FT, Pfeifer RM \& Maria IC (2005) Relações solos/ vegetação em área natural no Parque Estadual de Porto Ferreira, São Paulo. Revista do Instituto Florestal 17: 45-61.
Santos RM \& Vieira FA (2006) Florística e estrutura da comunidade arbórea de fragmentos de matas ciliares dos rios São Francisco, Cochá e Carinhanha, norte de Minas Gerais, Brasil. Revista Científica Eletrônica Engenharia Florestal 8: 1-18.

Souza JP, Araújo GM \& Haridasan M (2007) Influence of soil fertility on the distribution of tree species in a deciduous forest in the Triângulo Mineiro region of Brazil. Plant Ecology 191:253-263.

ter Braak CJF (1986) Canoniccal correspondence analysis: a new eigenvector technique for multivariate direct gradient analysis. Ecology 67: 1167-1179.

ter Braak CJF (1995) Ordination. In: Jongman RHG, ter Braak CJF \& van Tongeren OFR (eds.) Data analysis in community and landscape ecology. Cambridge University Press, Cambridge. 91-173p.

ter Braak CJF \& Prentice IC (1988) A theory of gradient analysis. Advances in Ecological Research 18: 271-317.

ter Braak CJF, \& Smilauer P (1988) Canoco - Reference manual and user's guide to Canoco for Windows: software for canonical community ordination. Microcomputer Power, Ithaca. 352p.

Veloso MDM, Nunes YRF, Azevedo IFP, Rodrigues PMS, Fernandes LA, Santos RM, Fernandes GW \& Pereira JAA (2014) Floristic and structural variations of the arboreal community in relation to soil properties in the Pandeiros river riparian forest, Minas Gerais, Brazil. Interciencia 39: 628-636.

Zar JH (1996) Biostatistical analysis. Prentice-Hall, New Jersey. $663 \mathrm{p}$. 